Pacific Journal of Mathematics

AN EVALUATION OF THE CONDITIONAL YEH-WIENER 


\section{AN EVALUATION OF THE CONDITIONAL YEH-WIENER INTEGRAL}

\section{Kun Soo Chang, Jae Moon Ahn and Joo Sup Chang}

Yeh obtained the conditional Wiener integral of

$$
\exp \left\{-\int_{0}^{t} V[x(u)] d u\right\}
$$

given $x(t)$ where $x$ is in Wiener space $C[0, t]$ and $V$ is a function on $\mathbf{R}^{1}$ satisfying certain conditions. In this paper we extend Yeh's result to the conditional Yeh-Wiener integral of $\exp \left\{-\int_{0}^{t} \int_{0}^{s} V[x(u, v)] d u d v\right\}$ given $x(s, t)$ where $x$ is in Yeh-Wiener space $C_{2}(Q)$ and $V$ is a nonnegative continuous function on $\mathbf{R}^{1}$ satisfying the condition

$$
\int_{\mathbf{R}^{1}} V(w) \cdot \exp \left\{-\frac{w^{2}}{2 s t}\right\} d m_{L}(w)<\infty .
$$

1. Introduction. Yeh recently derived inversion formulae for conditional expectations [5] and for conditional Wiener integrals [6]. He also evaluated some conditional Wiener integrals using these inversion formulae. In [2] and [3], they introduced the conditional Yeh-Wiener integral and extended some of Yeh's results for the conditional Wiener integrals to the conditional Yeh-Wiener integrals.

Here the probability space is the Yeh-Wiener measure space on the Yeh-Wiener space $C_{2}(Q)$ of the real valued continuous functions $x$ defined on $Q=[0, s] \times[0, t]$ for some fixed positive real numbers $s$ and $t$ such that $x(0, v)=x(u, 0)=0$ for all $0 \leq u \leq s$ and $0 \leq v \leq t$. In this paper we shall always denote $Q$ as a fixed above rectangle. Let $\left(C_{2}(Q)\right.$, $\mathscr{Y}, m_{y}$ ) be the Yeh-Wiener measure space. For a complete discussion of Yeh-Wiener measure space, see [7].

A real valued functional $F$ on $C_{2}(Q)$ is said to be Yeh-Wiener measurable if it is $\mathscr{Y}$-measurable. Its integral with respect to $m_{y}$ if it exists, is called its Yeh-Wiener integral which is denoted by $E^{y}(F)$. In this case we write

$$
E^{y}(F)=\int_{C_{2}(Q)} F(x) d m_{y}(x) .
$$

We say that $F$ is Yeh-Wiener integrable or $m_{y}$-integrable when the Yeh-Wiener integral of $F, E^{y}(F)$, exists and is finite. The Yeh-Wiener measurability and Yeh-Wiener integrability of a complex valued functional on $C_{2}(Q)$ are defined in terms of its real and imaginary parts. 
Let $X$ and $Y$ be the $\mathbf{R}^{n}$-valued and real valued Yeh-Wiener measurable functions on $C_{2}(Q)$, respectively, with $E^{y}(|Y|)<\infty$. Let $P_{X}$ be the probability distribution determined by $X$. By the conditional Yeh-Wiener integral of $Y$ given $X$ we mean the conditional expectation $E^{y}(Y \mid X)$ which is given as a function on the value space of $X$. Throughout this paper we shall be exclusively concerned with $X$ and $Y$ given by $X(x)=$ $x(s, t)$ and $Y(x)=\exp \left\{-\int_{Q} V[x(u, v)] d u d v\right\}$ for $x \in C_{2}(Q)$ in which $V$ is a nonnegative continuous function on $\mathbf{R}^{1}$ satisfying the condition

$$
\int_{\mathbf{R}^{1}} V(w) \cdot \exp \left\{-\frac{w^{2}}{2 s t}\right\} d m_{L}(w)<\infty
$$

where $m_{L}$ is the Lebesgue measure on $\mathbf{R}^{1}$.

The techniques of this paper are closely related to those of paper [6] of Yeh, but for the evaluation of the conditional Yeh-Wiener integral we use slightly different techniques. In Theorem 2.1 we evaluate the conditional Yeh-Wiener integral of $Y(x)$ given $X(x)$ which is the extension of Yeh's result [6; Theorem 5]. The proof of Theorem 2.1 is simpler than that of Yeh. To do this we will use the following Proposition which comes from [3; Theorem 3.5].

Proposition 1.1. Let $X$ and $Y$ be measurable transformations of $\left(C_{2}(Q), \mathscr{Y}\right)$ into $\left(\mathbf{R}^{1}, \mathscr{B}\left(\mathbf{R}^{1}\right)\right)$ with $E^{y}(|Y|)<\infty$. Assume that $P_{X}$ is absolutely continuous with respect to $m_{L}$ on $\left(\mathbf{R}^{1}, \mathscr{B}\left(\mathbf{R}^{1}\right)\right)$ and $E^{y}\left(e^{i u X} Y\right)$ is a $m_{L}$-integrable function of $u$ on $\mathbf{R}^{1}$. Then there exists a version of $E^{y}[Y \mid X]\left(d P_{X} / d m_{L}\right)$ such that for $\xi \in \mathbf{R}^{1}$,

$$
E^{y}[Y \mid X](\xi) \frac{d P_{X}}{d m_{L}}(\xi)=\frac{1}{2 \pi} \int_{\mathbf{R}^{\prime}} e^{-i u \xi} E^{y}\left(e^{i u X} Y\right) d m_{L}(u) .
$$

2. The conditional Yeh-Wiener integral of

$$
\exp \left\{-\int_{0}^{t} \int_{0}^{s} V[x(u, v)] d u d v\right\}
$$

given $x(s, t)$.

THEOREM 2.1. For some fixed positive real numbers $s$ and $t$, let

$$
\begin{aligned}
& X_{(s, t)}(x)=x(s, t) \text { and } \\
& Y_{(s, t)}(x)=\exp \left\{-\int_{0}^{t} \int_{0}^{s} V[x(u, v)] d u d v\right\}
\end{aligned}
$$

for $x \in C_{2}(Q)$ where $V$ is a nonnegative continuous function on $\mathbf{R}^{1}$ satisfying the condition

$$
\int_{\mathbf{R}^{1}} V(w) \exp \left\{-\frac{w^{2}}{2 s t}\right\} d m_{L}(w)<\infty
$$


for every $s$ and $t$ in $(0, \infty)$. Then the conditional Yeh-Wiener integral of $Y_{(s, t)}$ given by $X_{(s, t)}$ is

$$
\begin{aligned}
& E^{y}\left[Y_{(s, t)} \mid X_{(s, t)}\right](\zeta) \\
& =1-\int_{Q}\left[\int_{\mathbf{R}^{3}} \sqrt{\frac{s t}{(s-u)(t-v)}}\right. \\
& \quad \times\left\{\exp \left(-\frac{\left(\zeta-u_{21}-u_{12}+u_{11}\right)^{2}}{2(s-u)(t-v)}+\frac{\zeta^{2}}{2 s t}\right)\right\} \\
& \quad \times\left\{V\left(u_{11}\right) E^{y}\left[Y_{(u, v)} \mid X\right]\left(u_{11}, u_{12}, u_{21}\right)\right. \\
& \left.\left.-E^{y}\left[Z_{(u, v)} Y_{(u, v)} \mid X\right]\left(u_{11}, u_{12}, u_{21}\right)\right\} d P_{X}\left(u_{11}, u_{12}, u_{21}\right)\right] d m_{L}(u, v)
\end{aligned}
$$

where $x(s, t)=\zeta \in \mathbf{R}^{1}$,

$$
\begin{aligned}
Z_{(u, v)}(x) & =\int_{0}^{v} V[x(u, r)] d r \int_{0}^{u} V[x(q, v)] d q, \\
X(x) & =\left(X_{(u, v)}(x), X_{(u, t)}(x), X_{(s, v)}(x)\right)
\end{aligned}
$$

for $x \in C_{2}(Q)$, and

(2.6) $d P_{X}\left(u_{11}, u_{12}, u_{21}\right)=\left\{(2 \pi)^{3} u^{2} v_{1}^{2}(s-u)(t-v)\right\}^{-1 / 2}$

$$
\times \exp \left\{-\frac{u_{11}^{2}}{2 u v}-\frac{\left(u_{12}-u_{11}\right)^{2}}{2 u(t-v)}-\frac{\left(u_{21}-u_{11}\right)^{2}}{2(s-u) v}\right\} d m_{L}\left(u_{11}, u_{12}, u_{21}\right) .
$$

REMARK. The existence of $V$ on (2.2) follows if $V$ satisfies the order of growth condition

$$
V(w)=O\left(\exp \left\{w^{2-\delta}\right\}\right) \quad \text { as } w \rightarrow \pm \infty
$$

for some $\delta \in(0,2)$. Under (2.2), if we define

$$
\phi((s, t))=\frac{1}{\sqrt{2 \pi s t}} \int_{\mathbf{R}^{1}} V(w) \exp \left\{-\frac{w^{2}}{2 s t}\right\} d m_{L}(w)
$$

for $(s, t) \in(0, \infty)^{2}$, then $\phi$ is a nonnegative continuous function on $(0, \infty)^{2}$ and furthermore

$$
\lim _{(s, t) \rightarrow(\sigma, \tau)} \phi(s, t)=V(o) \quad \text { for }(\sigma, \tau) \in[0, \infty)^{2}-(0, \infty)^{2} .
$$


Let us define

$$
\phi(\sigma, \tau)=\lim _{(s, t) \rightarrow(\sigma, \tau)} \phi(s, t)
$$

for $(\sigma, \tau) \in[0, \infty)^{2}-(0, \infty)^{2}$ so that $\phi$ is continuous on $[0, \infty)^{2}$.

LEMMA 2.1. For $0<u \leq s$ and $0<v \leq t$,

$$
\begin{array}{r}
E^{v}\left[e^{i w(x(s, t)-x(s, v)-x(u, t)+x(u, v))}\right]= \\
\exp \left\{-\frac{(s-u)(t-v)}{2} w^{2}\right\} \\
\text { for } x \in C_{2}(Q) \text { and } w \in \mathbf{R}^{1} .
\end{array}
$$

The lemma can be followed from the fact that the left-hand side of (2.9) is the characteristic function of the random variable $x(s, t)-x(s, v)$ $-x(u, t)+x(u, v)$ whose probability distribution is the normal distribution with mean 0 and variance $(s-u)(t-v)$.

Proof of Theorem 2.1. We can easily obtain that $X_{(s, t)}$ and $Y_{(s, t)}$ are measurable transformations of $\left(C_{2}(Q), \mathscr{Y}\right)$ into $\left(\mathbf{R}^{1}, \mathscr{B}\left(\mathbf{R}^{1}\right)\right)$, with $E^{y}(|Y|)$ $<\infty$. Now

$$
\begin{aligned}
\frac{\partial^{2}}{\partial u \partial v}[ & \left.\exp \left\{-\int_{0}^{v} \int_{0}^{u} V[x(q, r)] d q d r\right\}\right] \\
= & \exp \left\{-\int_{0}^{v} \int_{0}^{u} V[x(q, r)] d q d r\right\} \\
& \times\left\{\int_{0}^{v} V[x(u, r)] d r \cdot \int_{0}^{u} V[x(q, v)] d q-V[x(u, v)]\right\},
\end{aligned}
$$

thus we have by (2.1)

$$
\begin{aligned}
& Y_{(s, t)}(x)=1+\int_{Q}\left\{\exp \left(-\int_{0}^{v} \int_{0}^{u} V[x(q, r)] d q d r\right)\right\} \\
& \times\left\{\int_{0}^{v} V[x(u, r)] d r \int_{0}^{u} V[x(q, v)] d q-V[x(u, v)]\right\} d m_{L}(u, v) .
\end{aligned}
$$

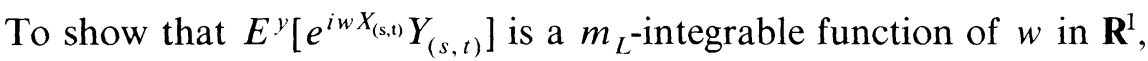
let

$$
E^{y}\left[e^{i w X_{(s, t)}} Y_{(s, t)}\right]=E^{y}\left[e^{i w x(s, t)}\right]-J_{1}(s, t)+J_{2}(s, t)
$$

where

(2.13) $J_{1}(s, t)$

$$
\begin{aligned}
=E^{y}\left[e^{i w x(s, t)}\right. & \int_{Q} V[x(u, v)] \\
& \left.\times \exp \left\{-\int_{0}^{v} \int_{0}^{u} V[x(q, r)] d q d r\right\} d m_{L}(u, v)\right]
\end{aligned}
$$


and

(2.14) $J_{2}(s, t)$

$$
\begin{array}{r}
=E^{y}\left[e^{i \omega x(s, t)} \int_{Q}\left\{\int_{0}^{v} V[x(u, r)] d r \int_{0}^{u} V[x(q, v)] d q\right\}\right. \\
\left.\times \exp \left\{-\int_{0}^{v} \int_{0}^{u} V[x(q, r)] d q d r\right\} d m_{L}(u, v)\right] .
\end{array}
$$

Then we can have

$$
E^{y}\left[e^{l w x(s, t)}\right]=\frac{1}{\sqrt{2 \pi s t}} \int_{\mathbf{R}^{1}} e^{i w \xi} e^{-\xi^{2} / 2 s t} d m_{L}(\xi)=e^{-(s t / 2) w^{2}}
$$

by the basic Yeh-Wiener integration formula and the formula

$$
\int_{\mathbf{R}^{1}} \exp \left\{-\left(a \xi^{2}+b \xi\right)\right\} d m_{L}(\xi)=\sqrt{\frac{\pi}{a}} \exp \left\{\frac{b^{2}}{4 a}\right\}
$$

for $a>0$ and real or imaginary $b$. Thus

$$
\int_{\mathbf{R}^{1}}\left|E^{y}\left[e^{i w x(s, t)}\right]\right| d m_{L}(w)=\sqrt{\frac{2 \pi}{s t}}<\infty .
$$

To interchange the order of the Yeh-Wiener integral and the integral with respect to $d m_{L}(u, v)$ on $Q$ in (2.13), note that

$$
\left|e^{i w x(s, t)} V[x(u, v)] \exp \left\{-\int_{0}^{v} \int_{0}^{u} V[x(q, r)] d q d r\right\}\right| \leq V[x(u, v)]
$$

for $((u, v), x) \in Q \times C_{2}(Q)$ and note that by (2.7) and the continuity of $\phi$ on $[0, \infty)^{2}$,

$$
\int_{Q} E^{y}[V(x(u, v))] d m_{L}(u, v)=\int_{Q} \phi(u, v) d m_{L}(u, v)<\infty .
$$

By the Fubini Theorem we have

(2.18) $J_{1}(s, t)$

$$
\begin{aligned}
=\int_{Q} E^{y}[ & e^{i w x(s, t)} V[x(u, v)] \\
& \left.\times \exp \left\{-\int_{0}^{v} \int_{0}^{u} V[x(q, r)] d q d r\right\}\right] d m_{L}(u, v) .
\end{aligned}
$$

Since

$$
\{x(s, t)-x(s, v)-x(u, t)+x(u, v),\{x(s, v), x(u, t), x(q, r)\}\}
$$


is an independent system of random variables on $\left(C_{2}(Q), \mathscr{Y}, m_{y}\right)$ for every $(q, r) \in[0, u] \times[0, v]$, we can have by Lemma 2.1,

$$
\begin{aligned}
J_{1}(s, t)= & \int_{Q} \exp \left\{-\frac{(s-u)(t-v)}{2} w^{2}\right\} \\
& \times E^{y}\left[e^{i w(x(s, v)+x(u, t)-x(u, v))} V[x(u, v)]\right. \\
& \left.\quad \times \exp \left\{-\int_{0}^{v} \int_{0}^{u} V[x(q, r)] d q d r\right\}\right] d m_{L}(u, v) .
\end{aligned}
$$

Let $X$ be a three dimensional random vector on $\left(C_{2}(Q), \mathscr{Y}, m_{y}\right)$ given by

$$
X(x)=\left(X_{1}(x), X_{2}(x), X_{3}(x)\right)
$$

where $X_{1} \equiv X_{(u, v)}, X_{2} \equiv X_{(u, t)}$, and $X_{3} \equiv X_{(s, v)}$. Let $Y_{1} \equiv Y_{(u, v)}$. Then the regular conditional distribution of $Y_{1}$ given $X, P\left(Y_{1} \mid X\right)$, exists since $Y_{1}$ is a real valued random variable. With fixed $w \in \mathbf{R}^{1}$ consider a complex valued function $f$ on $\mathbf{R}^{3} \times \mathbf{R}^{1}$ defined by

$$
f\left(\left(\xi_{1}, \xi_{2}, \xi_{3}\right), \eta\right)=e^{i w\left(\xi_{3}+\xi_{2}-\xi_{1}\right)} V\left(\xi_{1}\right) \eta
$$

for $\xi=\left(\xi_{1}, \xi_{2}, \xi_{3}\right) \in \mathbf{R}^{3}$ and $\eta \in \mathbf{R}^{1}$. By Proposition 2 and Proposition 1 in [5], we have

$$
\begin{aligned}
& E^{y}\left[e^{i w(x(s, v)+x(u, t)-x(u, v))} V[x(u, v)]\right. \\
&\left.\times \exp \left\{-\int_{0}^{v} \int_{0}^{u} V[x(q, r)] d q d r\right\}\right] \\
&= \int_{\mathbf{R}^{3}}\left\{\int_{\mathbf{R}^{1}} e^{i w\left(\xi_{3}+\xi_{2}-\xi_{1}\right)} V\left(\xi_{1}\right) \eta P\left(Y_{1} \mid X\right)(d \eta, \xi)\right\} d P_{X}(\xi) \\
&= \int_{\mathbf{R}^{3}} e^{i w\left(\xi_{3}+\xi_{2}-\xi_{1}\right)} V\left(\xi_{1}\right) E^{y}\left(Y_{1} \mid X\right)(\xi) d P_{x}(\xi) \\
&= \int_{\mathbf{R}^{3}} e^{i w\left(u_{21}+u_{12}-u_{11}\right)} V\left(u_{11}\right) E^{y}\left(Y_{1} \mid X\right)\left(u_{11}, u_{12}, u_{21}\right) \\
& d P_{X}\left(u_{11}, u_{12}, u_{21}\right)
\end{aligned}
$$

where

(2.23) $d P_{X}\left(u_{11}, u_{12}, u_{21}\right)=\left\{(2 \pi)^{3} u^{2} v^{2}(s-u)(t-v)\right\}^{-1 / 2}$

$$
\times \exp \left\{-\frac{u_{11}^{2}}{2 u v}-\frac{\left(u_{12}-u_{11}\right)^{2}}{2 u(t-v)}-\frac{\left(u_{21}-u_{11}\right)^{2}}{2(s-u) v}\right\} d m_{L}\left(u_{11}, u_{12}, u_{21}\right) \text {. }
$$


By (2.19) and (2.22) we can obtain

$$
\begin{aligned}
J_{1}(s, t)= & \int_{Q} \exp \left\{-\frac{(s-u)(t-v)}{2} w^{2}\right\} \\
& \times\left[\int_{\mathbf{R}^{3}} e^{i w\left(u_{21}+u_{12}-u_{11}\right)} V\left(u_{11}\right)\right. \\
& \left.\times E^{y}\left(Y_{1} \mid X\right)\left(u_{11}, u_{12}, u_{21}\right) d P_{X}\left(u_{11}, u_{12}, u_{21}\right)\right] d m_{L}(u, v) .
\end{aligned}
$$

To show that $J_{1}(s, t)$ is integrable, observe that

$$
\begin{aligned}
& \left|V\left(u_{11}\right) E^{y}\left(Y_{1} \mid X\right)\left(u_{11}, u_{12}, u_{21}\right) e^{i w\left(u_{21}+u_{12}-u_{11}\right)} e^{-((s-u)(t-v) / 2) w^{2}}\right| \\
& \leq V\left(u_{11}\right) E^{y}\left(Y_{1} \mid X\right)\left(u_{11}, u_{12}, u_{21}\right) e^{-((s-u)(t-v) / 2) w^{2}}
\end{aligned}
$$

for $\left(\left(u_{11}, u_{12}, u_{21}\right),(u, v)\right) \in \mathbf{R}^{3} \times Q$. Let $\pi_{1}$ be a function from $\mathbf{R}^{3}$ to $\mathbf{R}^{1}$ defined by $\pi_{1}\left(u_{11}, u_{12}, u_{21}\right)=u_{11}$. Then $X_{1}=\pi_{1} \circ X$. Thus by Proposition 3 in [5] and (2.7) we have

$$
\begin{array}{r}
\int_{\mathbf{R}^{3}} V\left(u_{11}\right) E^{y}\left(Y_{1} \mid X\right)\left(u_{11}, u_{12}, u_{21}\right) d P_{X}\left(u_{11}, u_{12}, u_{21}\right) \\
=E^{y}\left[\left(V \circ X_{1}\right) Y_{1}\right] \leq E^{y}[V(x(u, v))]=\phi(u, v) .
\end{array}
$$

Now

$$
\int_{Q} \phi(u, v) e^{-((s-u)(t-v) / 2) w^{2}} d m_{L}(u, v)=K e^{-(s t / 2) w^{2}}
$$

where

$$
K \equiv \int_{Q} \phi(u, v) e^{((s v+u t-u v) / 2) w^{2}} d m_{L}(u, v)<\infty
$$

by the continuity of $\phi$ on $[0, \infty)^{2}$. Thus $J_{1}(s, t)$ is integrable since

$$
\int_{\mathbf{R}^{1}}\left|J_{1}(s, t)\right| d m_{L}(w) \leq K \int_{\mathbf{R}^{1}} e^{-(s t / 2) w^{2}} d m_{L}(w)<\infty .
$$

To interchange the order of the integrals in (2.14) note that

$$
\begin{aligned}
& \mid e^{i w x(s, t)}\left\{\int_{0}^{v} V[x(u, r)]\right.\left.d r \int_{0}^{u} V[x(q, v)] d q\right\} \\
& \times \exp \left\{-\int_{0}^{v} \int_{0}^{u} V[x(q, r)] d q d r\right\} \mid \\
& \leq \int_{0}^{v} V[x(u, r)] d r \cdot \int_{0}^{u} V[x(q, v)] d q
\end{aligned}
$$

for $x \in C_{2}(Q)$ and

$$
\int_{Q} E^{y}\left[\int_{0}^{v} V[x(u, r)] d r \cdot \int_{0}^{u} V[x(q, v)] d q\right] d m_{L}(u, v) \leq M s t<\infty
$$


for some $M>0$ since $V \circ x$ is a continuous function. Thus from (2.14) we obtain

$$
\begin{aligned}
& J_{2}(s, t)=\int_{Q} \exp \left\{-\frac{(s-u)(t-v)}{2} w^{2}\right\} \\
& \quad \times E^{y}\left[e^{i w(x(s, v)+x(u, t)-x(u, v))}\right. \\
& \quad \times\left\{\int_{0}^{v} V[x(u, r)] d r \int_{0}^{u} V[x(q, v)] d q\right\} \\
& \left.\quad \times \exp \left\{-\int_{0}^{v} \int_{0}^{u} V[x(q, r)] d q d r\right\}\right] d m_{L}(u, v)
\end{aligned}
$$

by the Fubini Theorem and the same way as in (2.19). Let $X$ be given as in (2.20) and let $Y_{1}=Y_{(u, v)}$. Note that $E^{y}\left(\left|Y_{1}\right|\right)<\infty$. Let

$$
Z_{1}(x)=Z_{(u, v)}(x)=\int_{0}^{v} V[x(u, r)] d r \int_{0}^{u} V[x(q, v)] d q
$$

for $x \in C_{2}(Q)$. Then it is obvious that $Z_{1}$ is Yeh-Wiener measurable and Yeh-Wiener integrable on $C_{2}(Q)$. Put $F_{1} \equiv Z_{1} Y_{1}$. Then $F_{1}$ is a real valued random variable on $\left(C_{2}(Q), \mathscr{Y}, m_{y}\right)$ with $E^{y}\left(\left|F_{1}\right|\right)<\infty$. For fixed $w \in \mathbf{R}^{1}$ consider a complex valued function $g$ on $\mathbf{R}^{3} \times \mathbf{R}^{1}$ defined by

$$
g\left(\left(\xi_{1}, \xi_{2}, \xi_{3}\right), \eta\right)=e^{i w\left(\xi_{3}+\xi_{2}-\xi_{1}\right)} \eta
$$

for $\xi=\left(\xi_{1}, \xi_{2}, \xi_{3}\right) \in \mathbf{R}^{3}$ and $\eta \in \mathbf{R}^{1}$. Applying Proposition 2 and Proposition 1 in [5] to the real and imaginary parts of $g$ we have by (2.1),

$$
\begin{array}{r}
E^{y}\left[e^{i w(x(s, v)+x(u, t)-x(u, v))}\left\{\int_{0}^{v} V[x(u, r)] d r \int_{0}^{u} V[x(q, v)] d q\right\}\right. \\
\left.\times \exp \left\{-\int_{0}^{v} \int_{0}^{u} V[x(q, r)] d q d r\right\}\right] \\
=\int_{\mathbf{R}^{3}}\left\{\int_{\mathbf{R}^{1}} e^{i w\left(\xi_{3}+\xi_{2}-\xi_{1}\right)} \eta P\left(F_{1} \mid X\right)(d \eta, \xi)\right\} d P_{X}(\xi) \\
=\int_{\mathbf{R}^{3}} e^{i w\left(u_{21}+u_{12}-u_{11}\right)} E^{y}\left(F_{1} \mid X\right)\left(u_{11}, u_{12}, u_{21}\right) d P_{X}\left(u_{11}, u_{12}, u_{21}\right)
\end{array}
$$

where $d P_{X}\left(u_{11}, u_{12}, u_{21}\right)$ is given as in (2.23). By (2.27) and (2.28) we have

$$
\begin{aligned}
& J_{2}(s, t)=\int_{Q}\left\{\exp \left(-\frac{(s-u)(t-v)}{2} w^{2}\right)\right\} \\
& \times\left\{\int_{\mathbf{R}^{3}} e^{i w\left(u_{21}+u_{12}-u_{11}\right)} E^{y}\left(F_{1} \mid X\right)\left(u_{11}, u_{12}, u_{21}\right) d P_{X}\left(u_{11}, u_{12}, u_{21}\right)\right\} \\
& d m_{L}(u, v) .
\end{aligned}
$$


To show that $J_{2}(s, t)$ is integrable, observe that

$$
\begin{aligned}
& \left|E^{y}\left(F_{1} \mid X\right)\left(u_{11}, u_{12}, u_{21}\right) e^{i w\left(u_{21}+u_{12}-u_{11}\right)} e^{-((s-u)(t-v) / 2) w^{2}}\right| \\
& \quad \leq E^{y}\left(F_{1} \mid X\right)\left(u_{11}, u_{12}, u_{21}\right) e^{-((s-u)(t-v) / 2) w^{2}}
\end{aligned}
$$

for $\left(\left(u_{11}, u_{12}, u_{21}\right),(u, v)\right) \in \mathbf{R}^{3} \times Q$. Since the conditional Yeh-Wiener integral is $P_{X}$ integrable, we have

$$
N \equiv \int_{\mathbf{R}^{3}} E^{y}\left(F_{1} \mid X\right)\left(u_{11}, u_{12}, u_{21}\right) d P_{X}\left(u_{11}, u_{12}, u_{21}\right)<\infty .
$$

Thus

$$
\int_{Q} N e^{-((s-u)(t-v) / 2) w^{2}} d m_{L}(u, v)=N L e^{-(s t / 2) w^{2}}
$$

where

$$
L \equiv \int_{Q} e^{(s v+u t-u v / 2) w^{2}} d m_{L}(u, v)<\infty .
$$

Hence $J_{2}(s, t)$ is integrable since

$$
\text { (2.30) } \int_{\mathbf{R}^{1}}\left|J_{2}(s, t)\right| d m_{L}(w) \leq N L \int_{\mathbf{R}^{1}} e^{-(s t / 2) w^{2}} d m_{L}(w)<\infty .
$$

Therefore by (2.12), (2.17), (2.26) and (2.30), we have that $E^{v}\left[e^{i w X_{(s, t)}} Y_{(s, t)}\right]$ is a $m_{L^{-}}$-integrable function of $w$ on $\mathbf{R}^{1}$ and thus, by Proposition 1.1, there exists a version of

$$
E^{y}\left[Y_{(s, t)} \mid X_{(s, t)}\right] \frac{d P_{X_{(s, t)}}}{d m_{L}}
$$

such that

$$
\text { (2.31) } \begin{aligned}
E^{y} & {\left[Y_{(s, t)} \mid X_{(s, t)}\right](\zeta) \frac{d P_{X_{(s, t)}}}{d m_{L}}(\zeta) } \\
& =\frac{1}{2 \pi} \int_{\mathbf{R}^{1}} e^{-i w \zeta} E^{y}\left[e^{i w X_{(s, t)}} Y_{(s, t)}\right] d m_{L}(w) \\
& =\frac{1}{2 \pi} \int_{\mathbf{R}^{1}} e^{-i w \zeta}\left\{E^{y}\left[e^{i w x(s, t)}\right]-J_{1}(s, t)+J_{2}(s, t)\right\} d m_{L}(w)
\end{aligned}
$$

by (2.12). To evaluate the above integral first note that

$$
\frac{1}{2 \pi} \int_{\mathbf{R}^{1}} e^{-i w \zeta} E^{y}\left[e^{i w x(s, t)}\right] d m_{L}(w)=\frac{1}{\sqrt{2 \pi s t}} \exp \left\{-\frac{\zeta^{2}}{2 s t}\right\}
$$


by (2.15) and (2.16). And also note that

$$
\begin{aligned}
& \frac{1}{2 \pi} \int_{\mathbf{R}^{1}} e^{-i w \zeta} J_{1}(s, t) d m_{L}(w) \\
& =\frac{1}{2 \pi} \int_{Q}\left\{\int_{\mathbf{R}^{3}} V\left(u_{11}\right) E^{y}\left(Y_{1} \mid X\right)\left(u_{11}, u_{12}, u_{21}\right)\right. \\
& \quad \times\left[\int_{R^{1}} e^{-i w\left(\zeta-u_{21}-u_{12}+u_{11}\right)}\right. \\
& \left.\left.\times e^{-((s-u)(t-v) / 2) w^{2}} d m_{L}(w)\right] d P_{X}\left(u_{11}, u_{12}, u_{21}\right)\right\} d m_{L}(u, v)
\end{aligned}
$$

by (2.24) and the Fubini Theorem. By (2.16) we can have

$$
\begin{aligned}
& \frac{1}{2 \pi} \int_{\mathbf{R}^{1}} e^{-i w\left(\zeta-u_{21}-u_{12}+u_{11}\right)-((s-u)(t-v) / 2) w^{2}} d m_{L}(w) \\
& \quad=\frac{1}{\sqrt{2 \pi(s-u)(t-v)}} \exp \left\{-\frac{\left(\zeta-u_{21}-u_{12}+u_{11}\right)^{2}}{2(s-u)(t-v)}\right\}
\end{aligned}
$$

Substituting (2.34) in (2.33) we obtain

$$
\begin{aligned}
& \frac{1}{2 \pi} \int_{\mathbf{R}^{1}} e^{-i w \zeta} J_{1}(s, t) d m_{L}(w) \\
= & \int_{Q}\left\{\int_{\mathbf{R}^{3}} V\left(u_{11}\right) E^{y}\left(Y_{1} \mid X\right)\left(u_{11}, u_{12}, u_{21}\right) \frac{1}{\sqrt{2 \pi(s-u)(t-v)}}\right. \\
& \left.\times \exp \left\{-\frac{\left(\zeta-u_{21}-u_{12}+u_{11}\right)^{2}}{2(s-u)(t-v)}\right\} d P_{X}\left(u_{11}, u_{12}, u_{21}\right)\right\} d m_{L}(u, v) .
\end{aligned}
$$

Finally we can note that

(2.36) $\frac{1}{2 \pi} \int_{\mathbf{R}^{1}} e^{-i w \zeta} J_{2}(s, t) d m_{L}(w)$

$$
\begin{aligned}
=\int_{Q} & \left\{\int_{\mathbf{R}^{3}} E^{y}\left[F_{1} \mid X\right]\left(u_{11}, u_{12}, u_{21}\right) \frac{1}{\sqrt{2 \pi(s-u)(t-v)}}\right. \\
& \left.\times \exp \left\{-\frac{\left(\zeta-u_{21}-u_{12}+u_{11}\right)^{2}}{2(s-u)(t-v)}\right\} d P_{X}\left(u_{11}, u_{12}, u_{21}\right)\right\} d m_{L}(u, v)
\end{aligned}
$$

by (2.29), the Fubini Theorem and (2.34). Hence by (2.31), (2.32), (2.35) 
and (2.36) we have

$$
\text { (2.37) } \begin{aligned}
& E^{y}\left[Y_{(s, t)} \mid X_{(s, t)}\right](\zeta) \frac{d P_{X_{(s, t)}}(\zeta)}{d m_{L}} \\
= & \frac{1}{\sqrt{2 \pi s t}} \exp \left\{-\frac{\zeta^{2}}{2 s t}\right\} \\
& -\int_{Q}\left[\int _ { \mathbf { R } ^ { 3 } } \left\{V\left(u_{11}\right) E^{y}\left[Y_{1} \mid X\right]\left(u_{11}, u_{12}, u_{21}\right)\right.\right. \\
& \left.-E^{y}\left[F_{1} \mid X\right]\left(u_{11}, u_{12}, u_{21}\right)\right\} \frac{1}{\sqrt{2 \pi(s-u)(t-v)}} \\
& \left.\times \exp \left\{-\frac{\left(\zeta-u_{21}-u_{12}+u_{11}\right)^{2}}{2(s-u)(t-v)}\right\} d P_{X}\left(u_{11}, u_{12}, u_{21}\right)\right] d m_{L}(u, v) .
\end{aligned}
$$

Since

$$
\frac{d P_{X_{(s, t)}}}{d m_{L}}(\zeta)=\frac{1}{\sqrt{2 \pi s t}} \exp \left\{-\frac{\zeta^{2}}{2 s t}\right\},
$$

we can have the desired result (2.3) from (2.37).

\section{REFERENCES}

[1] L. Breiman, Probability, Addison-Wesley, Reading, Mass., 1968.

[2] K. S. Chang and J. M. Ahn, Translation theorem for conditional Yeh-Wiener integrals, J. Korean Math. Soc., 20 (1983), 163-172.

[3] D. M. Chung and J. M. Ahn, Conditional Yeh-Wiener integrals, J. Korean Math. Soc., 20 (1983), 209-221.

[4] J. Yeh, Stochastic Processes and the Wiener Integral, Marcel Dekker, Inc., New York, 1973.

[5] _ Inversion of conditional expectations, Pacific J. Math., 52 (1974), 631-640.

[6] _ Inversion of conditional Wiener integrals, Pacific J. Math., 59 (1975), 623-638.

[7] Wiener measure in a space of functions of two variables, Trans. Amer. Math. Soc., 95 (1960), 433-450.

Received March 19, 1985 and in revised form July 5, 1985. Research was partially supported by Korea Science and Engineering Foundation Grant.

YONSEI UNIVERSITY

SEOUL 120, KOREA

KONKUK UNIVERSITY

SEOUL 133, KOREA

AND

HANYANG UNIVERSITY

SEOUL 133, KOREA 



\section{PACIFIC JOURNAL OF MATHEMATICS EDITORS}

\author{
V. S. VARADARAJAN \\ (Managing Editor) \\ University of California \\ Los Angeles, CA 90024 \\ HeRbert Clemens \\ University of Utah \\ Salt Lake City, UT 84112 \\ R. FINN \\ Stanford University \\ Stanford, CA 94305
}

HERMANN FLASCHKA

University of Arizona

Tucson, AZ 85721

RAMESH A. GANGOLLI

University of Washington

Seattle, WA 98195

VAUGHAN F. R. JONES

University of California

Berkeley, CA 94720

ROBION KIRBY

University of California

Berkeley, CA 94720
C. C. MOORE

University of California

Berkeley, CA 94720

H. SAMELSON

Stanford University

Stanford, CA 94305

HAROLD STARK

University of California, San Diego

La Jolla, CA 92093

\section{ASSOCIATE EDITORS}
R. Arens
E. F. BECKENBACH
B. H. NEUMANN
F. WOLF
K. YoshIDA (1906-1982)

\section{SUPPORTING INSTITUTIONS}

UNIVERSITY OF ARIZONA

UNIVERSITY OF BRITISH COLUMBIA

CALIFORNIA INSTITUTE OF TECHNOLOGY

UNIVERSITY OF CALIFORNIA

MONTANA STATE UNIVERSITY

UNIVERSITY OF NEVADA, RENO

NEW MEXICO STATE UNIVERSITY

OREGON STATE UNIVERSITY
UNIVERSITY OF OREGON

UNIVERSITY OF SOUTHERN CALIFORNIA

TANFORD UNIVERSITY

UNIVERSITY OF HAWAII

UNIVERSITY OF TOKYO

UNIVERSITY OF UTAH

WASHINGTON STATE UNIVERSITY

UNIVERSITY OF WASHINGTON 


\section{Pacific Journal of Mathematics}

Vol. 124, No. $1 \quad$ May, 1986

Kinetsu Abe and Martin Andrew Magid, Relative nullity foliations and indefinite isometric immersions

Erik P. van den Ban, A convexity theorem for semisimple symmetric

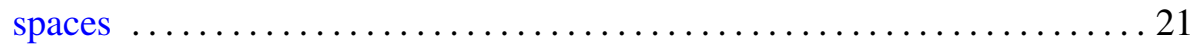

Bo Berndtsson and Thomas Joseph Ransford, Analytic multifunctions, the $\bar{\partial}$-equation, and a proof of the corona theorem .................. 57

Brian Boe and David H. Collingwood, Intertwining operators between

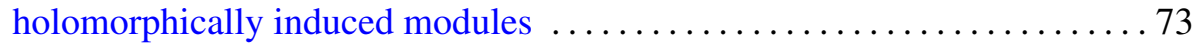

Giuseppe Ceresa and Alessandro Verra, The Abel-Jacobi isomorphism for

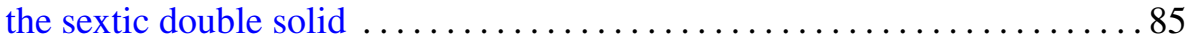

Kun Soo Chang, Jae Moon Ahn and Joo Sup Chang, An evaluation of the conditional Yeh-Wiener integral ........................... 107

Charles Dale Frohman, Minimal surfaces and Heegaard splittings of the

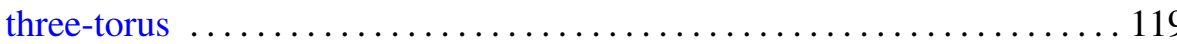

Robert M. Guralnick, Power cancellation of modules $\ldots \ldots \ldots \ldots \ldots \ldots 131$

Kenneth Hardy and Kenneth S. Williams, On the solvability of the Diophantine equation $d V^{2}-2 e V W-d W^{2}=1 \ldots \ldots \ldots \ldots \ldots \ldots \ldots \ldots \ldots$

Ray Alden Kunze and Stephen Scheinberg, Alternative algebras having

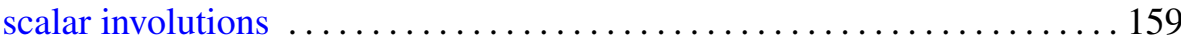

W. B. Raymond Lickorish and Kenneth Millett, The reversing result for the Jones polynomial $\ldots \ldots \ldots \ldots \ldots \ldots \ldots \ldots \ldots$

Guido Lupacciolu, A theorem on holomorphic extension of CR-functions

William Schumacher Massey and Lorenzo Traldi, On a conjecture of K. Murasugi

Dinakar Ramakrishnan, Spectral decomposition of $L^{2}(N \backslash \mathrm{GL}(2), \eta)$

Steven L. Sperber, On solutions of differential equations which satisfy certain algebraic relations 